\title{
Automatic Detection of Large Misregistrations of Multimodality Medical Images
}

\author{
C.E. Rodríguez-Carranza ${ }^{1,2}$ and M.H. Loew ${ }^{1,2}$ \\ 1 Department of Electrical and Computer Engineering, \\ 2 Institute for Medical Imaging and Image Analysis, \\ The George Washington University, \\ Washington DC 20052, USA \\ claudia, loew@seas.gwu . edu
}

\begin{abstract}
Before a retrospective registration algorithm can be used routinely in the clinic, methods must be provided for distinguishing between registration solutions that are clinically satisfactory and those that are not 1]. One approach is to rely on a human observer. Here, we present an algorithmic procedure that discriminates between badly misregistered pairs and those that are close to correct alignment. We found that a new goodness measure based on brain contours appears to identify misregistrations on the order of $15 \mathrm{~mm}$ or more of RMS error.
\end{abstract}

\section{Introduction and Design}

The lack of ground truth in the clinical setting makes it essential to find methodologies to assess automated retrospective registration techniques (which sometimes converge at clinically unsatisfactory values). Visual assessment has been used [21] to distinguish registrations that were clinically useful from those that were not. We would like to find alternative automatic or semiautomatic assessment techniques that, at the very least, reduce the number of bad registrations reaching the clinician. Therefore, we propose and demonstrate an algorithmic assessment technique for 3D CT-MR brain registration based on contours.

The measure of the goodness of registration used in this research was R1 the average fraction of MR contour voxels that were at a distance of zero or one (in the $\mathrm{x}-\mathrm{y}$ plane) from a CT contour voxel. $\mathrm{R} 1$ lies in the range $[0,1]$, and the larger R1 is, the closer the contours are. The aim is to use R1 to assign one of two possible labels to a registration result: "definitively bad" or "possibly good".

The measure was tested on CT, T1, and T2 images of three patients (practice set, patients 1 and 5) from the Vanderbilt study 3]. The registration error was defined as the RMS distance between the two sets of eight corner points of CT produced by its registration to MR using (1) the gold standard and (2) a given registration transformation.

To study the feasibility of R1 for assessing accuracy, we performed CT to $\mathrm{T} 1$ and $\mathrm{CT}$ to T2 registrations. A total of 800 misregistrations were generated per image pair per patient. The magnitudes of the resultant translational and rotational displacement vectors were confined to $0-8 \mathrm{~mm}$ and $0-8^{\circ}$, with corresponding RMS errors of $0-35 \mathrm{~mm}$. 


\section{Results and Discussion}

Figure 1 shows the results for the three patients $(801 \times 3=2403$ points per graph). Based on Wong et al. [2], we define registrations within both $3 \mathrm{~mm}$ and $4^{\circ}$ from ground truth to be good registrations (diamonds), and outside that range to be bad registrations (dots). Observe that for $\mathrm{R} 1=0.7$ most of the bad points with RMS $>15 \mathrm{~mm}$ could be identified. R1 is relatively insensitive to small rotations around the $X$ or $Y$ axes, so several bad points with RMS $>10 \mathrm{~mm}$ have a large value of $\mathrm{R} 1$; this is something that clearly needs to be improved. Points with RMS $<10 \mathrm{~mm}$ but $\mathrm{R} 1<0.5$ have large translational displacements, and therefore are real misregistrations.

How important to R1's usefulness is the accuracy of segmentation? The dependence is small because for registrations far from correct alignment the actual distance between contours will be much larger than the error induced by segmentation; work is underway on a general proof of this observation.
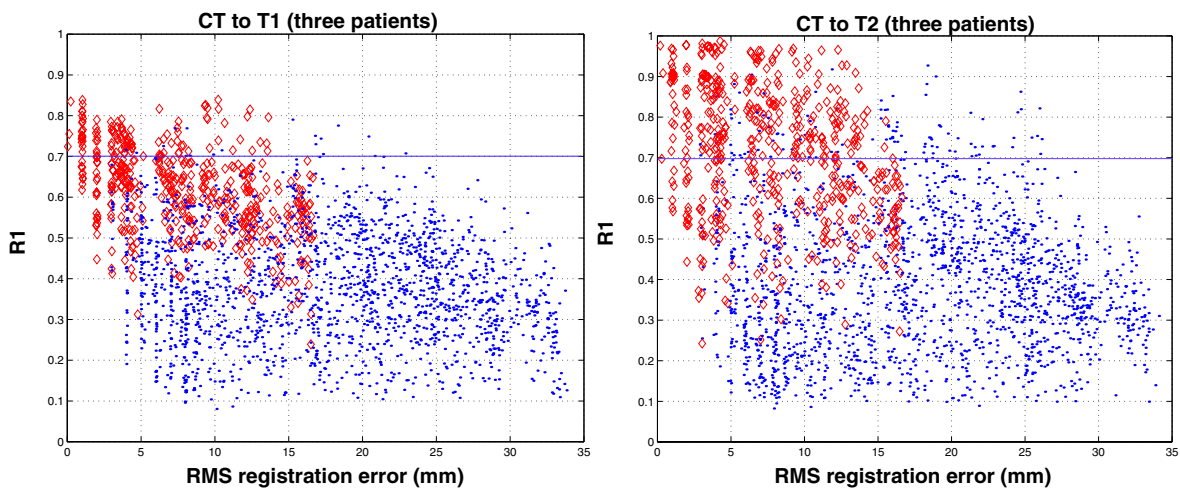

Fig. 1. Goodness of registration measure R1 against registration error.

\section{Conclusions}

We proposed a goodness measure based on brain contours as an aid to labeling registrations as either "definitively bad" or "possibly good". The results of the present study indicate that the use of R1 may be a good first step to reduce the number of badly registered images reaching the clinician.

\section{References}

1. Fitzpatrick, J.M., et al.: Visual assessment of the accuracy of retrospective registration of MR and CT images of the brain. IEEE TMI 17(4) (1998) 571-585

2. Wong, J.C.H., et al.: Evaluation of the limits of visual detection of image misregistration in a brain F-18 FDG PET-MRI study. Eur. J. of Nuc. Med. 24(6) (1997)

3. West, J., et al.: Comparison and evaluation of retrospective intermodality brain image registration techniques. JCAT 21(4) (1997) 554-566 